\title{
ON THE GENERAL DECAY STABILITY OF STOCHASTIC DIFFERENTIAL EQUATIONS WITH UNBOUNDED DELAY
}

\author{
Xuejing Meng and BaOJian Yin
}

\begin{abstract}
This work focuses on the general decay stability of nonlinear stochastic differential equations with unbounded delay. A Razumikhintype theorem is first established to obtain the moment stability but without almost sure stability. Then an improved edition is presented to derive not only the moment stability but also the almost sure stability, while existing Razumikhin-type theorems aim at only the moment stability. By virtue of the $M$-matrix techniques, we further develop the aforementioned Razumikhin-type theorems to be easily implementable. Two examples are given for illustration.
\end{abstract}

\section{Introduction}

In this paper, we consider the nonlinear stochastic differential equations with unbounded delay of the form

$$
d x(t)=f(t, x(t), y(t)) d t+g(t, x(t), y(t)) d w(t),
$$

where $y(t)=x(t-\delta(t)), \delta(t) \in C^{1}\left(\mathbb{R}_{+}, \mathbb{R}_{+}\right), f(t, x, y): \mathbb{R}_{+} \times \mathbb{R}^{n} \times \mathbb{R}^{n} \rightarrow \mathbb{R}^{n}$, $g(t, x, y): \mathbb{R}_{+} \times \mathbb{R}^{n} \times \mathbb{R}^{n} \rightarrow \mathbb{R}^{n \times m}$ are Borel measurable functions, and $w(t)$ is an $m$-dimensional Brownian motion.

Eq.(1.1) may be regarded as the stochastically perturbed systems of the following deterministic ordinary delay differential equations with unbounded delay

$$
d x(t)=f(t, x(t), y(t)) d t .
$$

For Eq.(1.2), relevant results or applications can be found, for example, in [5] or [6] for asymptotic estimation, variables' change in [7] and asymptotic behavior of the generalized pantograph differential equations in [9] or [12]. In both Eq.(1.2) and Eq.(1.1), there are some special systems, such as the equations with a power delay if $t-\delta(t)=t^{\gamma}, 0<\gamma<1, t \geq 1$, or the equations with a proportional delay if $t-\delta(t)=\lambda t, 0<\lambda<1, t \geq 0$. For

Received June 27, 2010; Revised October 21, 2011.

2010 Mathematics Subject Classification. 60H10, 34K20, 34K50.

Key words and phrases. stochastic delay differential equation, unbounded delay, Razumikhin-type theorem, stability, $M$-matrix. 
the latter, the equations are called (deterministic or stochastic) pantograph differential equations. Stochastic pantograph equations were studied in [2] for the polynomial asymptotic behavior, while linear stochastic pantograph equations were discussed in [1] for the polynomial stability.

The term general decay stability is also called the stability with general decay rate. To put it formally, we need to define the so-called decay functions.

Definition 1.1. A function $\psi: \mathbb{R} \rightarrow(0, \infty)$ is said to be a $\psi$-type function if it satisfies the following conditions:

(i) it is continuous and nondecreasing in $\mathbb{R}$ and continuously differentiable in $\mathbb{R}_{+}$;

(ii) $\psi(0)=1$ and $\psi(\infty)=\infty$;

(iii) let $\psi_{1}(t)=\psi^{\prime}(t) / \psi(t)$, then $\phi=\sup _{t \geq 0}\left[\psi_{1}(t)\right]<\infty$;

(iv) for any $t, s \geq 0, \psi(t) \leq \psi(s) \psi(t-s)$.

It is obvious that functions $\psi(t)=e^{\alpha t}$ and $\psi(t)=\left(1+t^{+}\right)^{\bar{\alpha}}$ for all $\alpha, \bar{\alpha}>0$ are $\psi$-type functions. If $\psi_{1}$ and $\psi_{2}$ are two $\psi$-type functions, then $\psi_{1} \psi_{2}$ is still a $\psi$-type function.

Then we can define the $p$-th moment $\psi$-type stable and almost surely $\psi$-type stable as follow:

and

$$
\limsup _{t \rightarrow \infty} \frac{\log \mathbb{E}|x(t)|^{p}}{\log \psi(t)}<0
$$

$$
\limsup _{t \rightarrow \infty} \frac{\log |x(t)|}{\log \psi(t)}<0 \quad \text { a.s. }
$$

It is obvious that $\psi$-type stability implies the exponential stability and the polynomial stability when $\psi(t)=e^{\alpha t}$ and $\psi(t)=\left(1+t^{+}\right)^{\bar{\alpha}}$ for all $\alpha, \bar{\alpha}>0$, respectively.

Stability is a central characteristic of the behavior in a (deterministic or stochastic) dynamical system. In general, time delay and system uncertainty are commonly encountered and are often sources of instability (see [11]). It is therefore interesting to consider the stability of Eq.(1.1) whose delay is unbounded. Moreover, unbounded delay systems are often more complicated than their bounded counterparts. Many methods which are valid for the former may be inefficient or impossible for the latter. Some classical techniques, such as Lyapunov direct methods in [13] or Razumikhin-type theorems in [14], can not be directly transferred to those of the unbounded delay cases.

As for the Lyapunov direct method, we refer the reader to see Arnold's book [3], Khasminskii's book [10], or the exponential stability in Mao [13] and Khasminskii-type theorems in Mao [23]. However, this method requires proper Lyapunov functionals to be found beforehand and hence it is sometimes difficult to do, especially in the case of stochastic functional differential equations (SFDEs). Obviously, Eq.(1.1) is one special class of SFDEs. To examine efficiently the stability of SFDEs, Mao [14] initiated Razumikhin-type method in 
1996. Then he extended it in neutral stochastic functional differential equations in Mao [15] in next year. In some sense, Razumikhin-type method has an advantage over the Lyapunov direct method, because the satisfied conditions for the former are usually weaker than the latter, that is, the conditions for the Lyapunov method usually hold in a whole function space while those for the Razumikhin-type method are much fewer. Along these lines, Mao and many other researchers acquired many known stability results in the bounded delay case; for example, see the stability analysis in Mao [16]. In the paper, our main efforts are taken to obtain the Razumikhin-type theorems in the unbounded delay case and to develop new techniques on the general decay stability.

As Mao stated in [21] that, generally speaking, the moment stability and the almost sure stability do not imply each other. To deal with the almost sure stability, there are several existing methods such as the exponential martingale inequality approach in Mao [20] and the LaSalle principle in his series' papers $[17,18,19]$ and an improved LaSalle method in Shen [25]. A well-known method is that combining the moment stability with the linear growth condition to obtain the almost sure stability, which is supported by Mao's other series' papers $[20,21,22,24]$. Much different from theirs, without the linear growth condition, we propose new method to make the $p$-th moment $\psi$-type stability imply the almost sure $\psi$-type stability in the unbounded delay case with the usual bounded delay as a special case.

The paper is arranged as follows. The next section begins with necessary notation and some assumptions in the paper. In Section 3, we establish a Razumikhin-type theorem and improve its key condition to obtain a new theorem on $p$-th moment $\psi$-type stability as well as the almost sure $\psi$-type stability. In Section 4, we apply these theorems to find some useful criteria in terms of $M$ matrix theory. As an application, we consider two two-dimensional examples to illustrate our theory in last section.

\section{Notations and assumptions}

Throughout this paper, unless otherwise specified, we use the following notation and definitions. Let $|\cdot|$ be the Euclidean norm in $\mathbb{R}^{n}$. If $A$ is a vector or matrix, its transpose is denoted by $A^{\mathrm{T}}$. If $A$ is a matrix, denote its trace norm by $|A|=\sqrt{\operatorname{trace}\left(A^{\mathrm{T}} A\right)}$. Let $\mathbb{R}_{+}=[0, \infty)$ and $\mathbb{R}_{++}=(0, \infty)$. Denote $\mathbb{R}_{++}^{n}=\left\{x \in \mathbb{R}^{n}: x_{i}>0, i=1, \ldots, n\right\}$ and $x \gg 0 \Leftrightarrow x \in \mathbb{R}_{++}^{n}$. For any $c=\left(c_{1}, \ldots, c_{n}\right)^{\mathrm{T}} \in \mathbb{R}^{n}$, let $\operatorname{diag}(c)=\operatorname{diag}\left(c_{i}\right)=\operatorname{diag}\left(c_{1}, c_{2}, \ldots, c_{n}\right)$ denote the $n \times n$ matrix with all elements zero except those on the diagonal which are $c_{1}, \ldots, c_{n}$. For simplicity, if $a, b \in \mathbb{R}$, we denote $a \wedge b=\min \{a, b\}$ and $a \vee b=\max \{a, b\}$. Let $a^{+}=a \vee 0$.

Let $(\Omega, \mathcal{F}, \mathbb{P})$ be a complete probability space with a filtration $\left\{\mathcal{F}_{t}\right\}_{t \geq 0}$ satisfying the usual conditions, that is, it is right continuous and increasing while $\mathcal{F}_{0}$ contains all $\mathbb{P}$-null sets. Let $w(t)$ be an $m$-dimensional Brownian motion defined on this probability space. 
Let $C^{2}\left(\mathbb{R}^{n} ; \mathbb{R}_{+}\right)$denote the family of all functions $V(x)$ from $\mathbb{R}^{n}$ to $\mathbb{R}_{+}$which are continuously twice differentiable, and define a function $\mathcal{L} V: \mathbb{R}_{+} \times \mathbb{R}^{n} \times \mathbb{R}^{n} \rightarrow$ $\mathbb{R}$ by

$$
\mathcal{L} V(t, x, y)=V_{x}(x) f(t, x, y)+\frac{1}{2} \operatorname{trace}\left[g^{\mathrm{T}}(t, x, y) V_{x x}(x) g(t, x, y)\right]
$$

for all $t \geq 0, x, y \in \mathbb{R}^{n}$, where

$$
V_{x}(x)=\left(\frac{\partial V(x)}{\partial x_{1}}, \frac{\partial V(x)}{\partial x_{2}}, \ldots, \frac{\partial V(x)}{\partial x_{n}}\right), \quad V_{x x}(x)=\left[\frac{\partial^{2} V(x)}{\partial x_{i} \partial x_{j}}\right]_{n \times n} .
$$

If $x(t)$ is a solution of Eq.(1.1), by the Itô formula,

$$
d V(x(t))=L V(x(t)) d t+V_{x}(x(t)) g(t, x(t), y(t)) d w(t),
$$

where

$$
L V(x(t))=\mathcal{L} V(t, x(t), y(t)), \quad y(t)=x(t-\delta(t)) .
$$

Let $C$ always represent a generic positive constant whose value may be different for different appearances so that $C+C=C$ and $C C=C$ is understood in an appropriate sense.

For convenience, let $\tau_{0}=\delta(0)$. Denote by $B C=B C\left(\left[-\tau_{0}, 0\right], \mathbb{R}^{n}\right)$ the family of all continuous functions from $\left[-\tau_{0}, 0\right]$ to $\mathbb{R}^{n}$ with the norm $\|\varphi\|=$ $\sup _{-\tau_{0} \leq \theta \leq 0}|\varphi(\theta)|$. If $\tau_{0}=0, B C=\mathbb{R}^{n}$.

Throughout the paper, we assume that Eq.(1.1) has a unique global solution which is denoted by $x(t)=x(t, \xi)$ on $t \geq \tau_{0}$ and $\xi \in B C$. As usual, we assume that $f(t, 0,0)=0, g(t, 0,0)=0$, which implies that Eq.(1.1) admits an equilibrium solution. Moreover, we impose the following standing assumptions:

Assumption 2.1. For any initial data $\xi \in B C, \xi \not \equiv 0$ and for some two constants $\alpha \geq 0$ and $p \geq 2$,

$$
\mathbb{E}\left[\|\xi\|^{\alpha+p}\right]<\infty
$$

Assumption 2.2. For the constant $\alpha \geq 0$,

$$
\left|x^{T} f(t, x, y)\right| \vee|g(t, x, y)|^{2} \leq C\left(1+|x|^{\alpha+2}+|y|^{\alpha+2}\right) \text {. }
$$

Assumption 2.3. For $c_{i}>0,1 \leq i \leq n$, there exists a function $V=$ $\sum_{i=1}^{n} c_{i}\left|x_{i}\right|^{p}$ such that for two constants $\mu>0, q \geq 1$, $(2.5)$

$$
\mathbb{E} L V(x(t)) \leq-\mu \psi_{1}(t) \mathbb{E} V(x(t)) \text {, whenever } \mathbb{E} V(y(t)) \leq q \psi^{\mu}(\delta(t)) \mathbb{E} V(x(t)) .
$$

Assumption 2.1 is the moment bounds for initial data, which is made as the basis on stability analysis. Assumption 2.2 implies the one-sided polynomial growth condition on $f$ and the polynomial growth condition on $g$. When $\alpha=0$, they are the classical one-sided linear growth condition and the linear growth condition, respectively, as in [8]. In addition, Assumption 2.3 is a usual assumption on the Razumikhin methods (see [14], [15] or [16]). 


\section{Razumikhin-type theorem and an improved edition}

In this section, for $p \geq 2$, we first establish a Razumikhin-type theorem on the $p$-th moment $\psi$-type stability of Eq.(1.1), then develop an improved edition to derive both $p$-th moment $\psi$-type stability and the almost sure $\psi$ type stability.

Firstly, we need a lemma whose proof is omitted since it can be easily completed by the standard estimate method as in [16], Ch.5.

Lemma 3.1. Under Assumptions 2.1 and 2.2, let $x(t)=x(t, \xi)(\xi \in B C)$ be a global solution of Eq.(1.1). Then for $\alpha \geq 0$ and $p \geq 2$,

$$
\mathbb{E}\left[\sup _{-\tau_{0} \leq s \leq t}|x(s)|^{\alpha+p}\right]<\infty .
$$

In the case of bounded delay, as some literature such as [21] or [22] put, the moment exponential stability and linear growth condition do imply the almost sure exponential stability. While in the case of unbounded delay, can this result be kept as well? Or under what conditions does the $p$-th moment $\psi$-type stability imply almost sure $\psi$-type stability? The following theorems address these questions.

Theorem 3.2. Under Assumptions 2.1-2.3, let

$$
S_{\mu}(\xi)=\sup _{-\tau_{0} \leq \theta \leq 0} \psi^{\mu}(\theta) V(\xi(\theta)) .
$$

Then the global solution $x(t)$ of Eq.(1.1) has the following properties:

$$
\begin{array}{r}
\mathbb{E} V(x(t)) \leq S_{\mu}(\xi) \psi^{-\mu}(t), \\
\mathbb{E}|x(t)|^{p} \leq C \psi^{-\mu}(t), \\
\limsup _{t \rightarrow \infty} \frac{\ln \mathbb{E}|x(t)|^{p}}{\ln \psi(t)} \leq-\mu .
\end{array}
$$

Proof. Obviously, (3.2) implies (3.3) and (3.3) implies (3.4). Therefore, we only need to prove (3.2). If we could prove $(3.2)$ for $\gamma \in(0, \mu)$, we would complete this proof. This is equivalent to proving that

$$
h(t):=\psi^{\gamma}(t) \mathbb{E} V(x(t)) \leq S_{\gamma}(\xi)
$$

for all $t \geq 0$.

First, when $t=0$, we have

$$
h(0)=\mathbb{E} V(\xi(0)) \leq S_{\gamma}(\xi) .
$$

Next we will show that (3.5) holds for all $t>0$. Otherwise, by the continuity of $h(t)$, there must exist a maximal $t_{0} \in \mathbb{R}_{+}$such that for all $t \in\left[0, t_{0}\right]$, $h(t) \leq S_{\gamma}(\xi)=h\left(t_{0}\right)$. Let $\Delta(t)=t-\delta(t)$, by the definition of $\psi$-type function, we have $\psi(t) \leq \psi(\delta(t)) \psi(\Delta(t))$. The situation is divided into two cases.

Case 1: $\Delta\left(t_{0}\right) \geq 0$. When $\Delta\left(t_{0}\right) \geq 0$, we know $\Delta\left(t_{0}\right) \in\left[0, t_{0}\right]$. Compute

$$
\mathbb{E} V\left(y\left(t_{0}\right)\right)=\mathbb{E} V\left(x\left(\Delta\left(t_{0}\right)\right)\right)=\psi^{-\gamma}\left(\Delta\left(t_{0}\right)\right) h\left(\Delta\left(t_{0}\right)\right)
$$




$$
\begin{aligned}
& \leq \psi^{-\gamma}\left(\Delta\left(t_{0}\right)\right) S_{\gamma}(\xi) \\
& =\psi^{-\gamma}\left(\Delta\left(t_{0}\right)\right) h\left(t_{0}\right) \\
& =\psi^{-\gamma}\left(\Delta\left(t_{0}\right)\right) \psi^{\gamma}\left(t_{0}\right) \mathbb{E} V\left(x\left(t_{0}\right)\right) \\
& \leq \psi^{\gamma}\left(\delta\left(t_{0}\right)\right) \mathbb{E} V\left(x\left(t_{0}\right)\right) \\
& \leq q \psi^{\mu}\left(\delta\left(t_{0}\right)\right) \mathbb{E} V\left(x\left(t_{0}\right)\right) .
\end{aligned}
$$

Case 2: $\quad \Delta\left(t_{0}\right)<0$. From $\Delta\left(t_{0}\right)<0$ we have

$$
\begin{aligned}
\mathbb{E} V\left(y\left(t_{0}\right)\right) & =\mathbb{E} V\left(\xi\left(\Delta\left(t_{0}\right)\right)\right) \\
& \leq \psi^{-\gamma}\left(\Delta\left(t_{0}\right)\right) S_{\gamma}(\xi) .
\end{aligned}
$$

Repeating the corresponding part of Case 1 yields

$$
\mathbb{E} V\left(y\left(t_{0}\right)\right) \leq q \psi^{\mu}\left(\delta\left(t_{0}\right)\right) \mathbb{E} V\left(x\left(t_{0}\right)\right) .
$$

By Assumption 2.3, we have

$$
\mathbb{E} L V\left(x\left(t_{0}\right)\right) \leq-\mu \psi_{1}\left(t_{0}\right) \mathbb{E} V\left(x\left(t_{0}\right)\right) .
$$

Note that $\mathbb{E} V\left(x\left(t_{0}\right)\right)>0$. Otherwise, $0=\mathbb{E} V\left(x\left(t_{0}\right)\right)=h\left(t_{0}\right)=S_{\gamma}(\xi)$. This contradicts $\xi \not \equiv 0$. Noting $\gamma<\mu$, when $t>t_{0}$ and $t$ is sufficiently close to $t_{0}$, we have

$$
\mathbb{E} L V(x(t)) \leq-\gamma \psi_{1}(t) \mathbb{E} V(x(t)) .
$$

By the Itô formula, we observe

$$
\begin{aligned}
h(T) & =h\left(t_{0}\right)+\int_{t_{0}}^{T} \psi^{\gamma}(t)\left[\mathbb{E} L V(x(t))+\gamma \psi_{1}(t) \mathbb{E} V(x(t))\right] d t \\
& \leq h\left(t_{0}\right)
\end{aligned}
$$

for $T>t_{0}$ and $T$ sufficiently near $t_{0}$. This contradicts the definition of $t_{0}$. So, (3.5) is confirmed for all $t \geq 0$.

In the above proof, the continuity of functions $h(t), \mathbb{E} V(x(t))$ and $\mathbb{E} L V(x(t))$ is regarded as a fact, which we will make up for its proof now. First, it is known that $V(x(t))$ and $L V(x(t))$ are almost surely pathwise continuous for all $t \geq 0$. Let $G(t)=\sup _{-\tau_{0} \leq s \leq t}|x(s)|^{\alpha+p}$. By Lemma 3.1, we have $\mathbb{E} G(t)<\infty$ for all $t \geq 0$. Since

$$
V(x(t)) \leq C|x(t)|^{p} \leq C[G(t)+1],
$$

by the Lebesgue dominated convergence theorem, we obtain that $\mathbb{E} V(x(t))$ is continuous and $h(t)$ is also continuous.

By Assumption 2.2, we can get

$$
\begin{aligned}
|\mathcal{L} V(t, x, y)| & \leq C\left(1+|x|^{\alpha+p}+|y|^{\alpha+p}\right), \\
|L V(x(t))| & \leq C[1+G(t)] .
\end{aligned}
$$

Applying the dominated convergence theorem again to derive that $\mathbb{E} L V(x(t))$ is continuous. This completes the proof. 
Obviously, there are some weaknesses in Theorem 3.2. Firstly, the key condition (2.5) is not easily tested. Moreover, like existing Razumikhin-type theorems (see [14], [15] or [16]), the almost sure stability can not be obtained. However, the following improved result will overcome these weaknesses and derive the almost sure stability without linear growth conditions.

Theorem 3.3. Let Assumptions 2.1 and 2.2 hold. Assume that there exist constants $a>b>0, \varepsilon>0$ and $c_{i}>0,1 \leq i \leq n$, such that for some function $V(x)=\sum_{i=1}^{n} c_{i}\left|x_{i}\right|^{p}(p \geq 2)$,

$$
\mathcal{L} V(t, x, y) \leq \psi_{1}(t)\left[-a V(x)+b V(y) \psi^{-\varepsilon}(\delta(t))\right] .
$$

Then for $\mu \in(0, \varepsilon]$, conclusions (3.2)-(3.4) of Theorem 3.2 still hold. Assume further that

$$
\left|V_{x}(x) g(t, x, y)\right| \leq C V(x),
$$

and there exists $\pi_{0} \in[0, \mu)$ such that for any $\pi>\pi_{0}$,

$$
\sum_{k=1}^{\infty} \psi^{-\pi}(k)<\infty
$$

then we have

$$
\limsup _{t \rightarrow \infty} \frac{\ln |x(t)|}{\ln \psi(t)} \leq-\frac{\mu-\pi_{0}}{p} \quad \text { a.s. }
$$

Proof. The proof will be divided into three steps.

Step 1. Noting $\psi(t)$ is increasing on $t$, for $\mu \in(0, \varepsilon]$ and $x(t)$ which satisfies

$$
\mathbb{E} V(y(t)) \leq q \psi^{\mu}(\delta(t)) \mathbb{E} V(x(t)),
$$

then by condition (3.6) we have

$$
\begin{aligned}
\mathbb{E} L V(x(t)) & \leq \psi_{1}(t)\left[-a \mathbb{E} V(x(t))+b \mathbb{E} V(y(t)) \psi^{-\varepsilon}(\delta(t))\right] \\
& \leq-a \psi_{1}(t) \mathbb{E} V(x(t))+b q \psi_{1}(t) \psi^{\mu}(\delta(t)) \psi^{-\varepsilon}(\delta(t)) \mathbb{E} V(x(t)) \\
& \leq-(a-b q) \psi_{1}(t) \mathbb{E} V(x(t)) .
\end{aligned}
$$

Let $\mu=\varepsilon \wedge(a-b)$, there surely exists $q \in[1, a / b)$ such that $\mu=a-b q>0$. Therefore, we have tested Assumption 2.3. Thus, by Theorem 3.2, conclusions (3.2)-(3.4) of Theorem 3.2 hold.

Step 2. Let $m(t)=\psi^{\mu}(t) V(x(t))$. We need to prove

$$
M(t):=\mathbb{E}\left[\sup _{t \leq s \leq t+1} m(s)\right] \leq C .
$$

By the Itô's formula, we have

$$
M(t)=\mathbb{E}\left\{\sup _{t \leq s \leq t+1}\left[m(t)+\int_{t}^{s} L m(r) d r+\int_{t}^{s} \psi^{\mu}(r) V_{x}(x(r)) g(r, x(r), y(r)) d w(r)\right]\right\}
$$




$$
\begin{aligned}
& =\mathbb{E} \sup _{t \leq s \leq t+1} m(t)+\mathbb{E}\left[\sup _{t \leq s \leq t+1} \int_{t}^{s} \operatorname{Lm}(r) d r\right] \\
& \quad+\mathbb{E}\left[\sup _{t \leq s \leq t+1} \int_{t}^{s} \psi^{\mu}(r) V_{x}(x(r)) g(r, x(r), y(r)) d w(r)\right] \\
& :=I_{1}+I_{2}+I_{3} .
\end{aligned}
$$

Then we will estimate $I_{1}, I_{2}$ and $I_{3}$, respectively.

Firstly, connecting the conclusion (3.3) with that

$$
I_{1}=\mathbb{E}\left[\sup _{t \leq s \leq t+1} \psi^{\mu}(t) V(x(t))\right]
$$

produces clearly that $I_{1} \leq C$.

Then by condition (3.6), we find that

$$
\begin{aligned}
I_{2} & =\mathbb{E}\left[\sup _{t \leq s \leq t+1} \int_{t}^{s} L m(r) d r\right] \\
& =\mathbb{E}\left[\sup _{t \leq s \leq t+1} \int_{t}^{s} \psi^{\mu}(r)\left[L V(x(r))+\mu \psi_{1}(r) V(x(r))\right] d r\right] \\
& \leq \mathbb{E} \int_{t}^{t+1} \psi^{\mu}(s) \psi_{1}(s)\left[b V(y(s)) \psi^{-\varepsilon}(\delta(s))+\mu V(x(s))\right] d s .
\end{aligned}
$$

Noting $\psi_{1}(s) \leq \phi$ and $\psi^{\mu}(s) \leq \psi^{\mu}(s-\delta(s)) \psi^{\mu}(\delta(s)) \leq \psi^{\mu}(s-\delta(s)) \psi^{\varepsilon}(\delta(s))$, we have

$$
I_{2} \leq \mathbb{E} \int_{t}^{t+1}\left[\phi b \psi^{\mu}(s-\delta(s)) V(y(s))+\phi \mu \psi^{\mu}(s) V(x(s))\right] d s .
$$

Using inequality (3.3) again, we get $I_{2} \leq C$.

Thirdly, applying the well-known Burkholder-Davis-Gundy inequality yields

$$
\begin{aligned}
I_{3} & =\mathbb{E}\left[\sup _{t \leq s \leq t+1} \int_{t}^{s} \psi^{\mu}(r) V_{x}(x(r)) g(r, x(r), y(r)) d w(r)\right] \\
& \leq C \mathbb{E}\left[\int_{t}^{t+1}\left|\psi^{\mu}(s) V_{x}(x(s)) g(s, x(s), y(s))\right|^{2} d s\right]^{1 / 2} .
\end{aligned}
$$

Recalling condition (3.7) and the inequality $\sqrt{a b} \leq(a+b) / 2, a, b>0$, we have

$$
\begin{aligned}
I_{3} & \leq C \mathbb{E}\left[\int_{t}^{t+1}\left|\psi^{\mu}(s) V(x(s))\right|^{2} d s\right]^{1 / 2} \\
& \leq C \mathbb{E}\left[\sup _{t \leq s \leq t+1} m(s) \int_{t}^{t+1} m(s) d s\right]^{1 / 2} \\
& \leq \frac{M(t)}{2}+C \mathbb{E} \int_{t}^{t+1} m(s) d s .
\end{aligned}
$$


Therefore, inserting all of these estimated bounds into Eq.(3.10) yields

$$
M(t) \leq C+\frac{1}{2} M(t)+C \mathbb{E} \int_{t}^{t+1} m(s) d s .
$$

Applying inequality (3.2) to get that $\mathbb{E} m(s) \leq C$, we have

$$
M(t) \leq C+\frac{1}{2} M(t)
$$

Since we know that $M(t)<\infty$ from Assumption 2.1, $M(t) \leq C$ as desired.

Step 3. Let $\pi \in\left(\pi_{0}, \mu\right)$ be arbitrary. By the Chebyshev inequality, we have

$$
\mathbb{P}\left(\sup _{k \leq t \leq k+1} m(t) \leq \psi^{\pi}(k)\right) \leq \psi^{-\pi}(k) M(k) \leq C \psi^{-\pi}(k), \quad k=1,2, \ldots
$$

Applying the Borel-Cantelli Lemma to get that for almost all $\omega \in \Omega$, sufficiently large $k$ and $k \leq t \leq k+1$, we have

$$
m(t) \leq \psi^{\pi}(k) \leq \psi^{\pi}(t) .
$$

This implies that for all $t \geq 0$,

$$
\psi^{\mu-\pi}(t) V(x(t)) \leq C .
$$

Therefore

$$
\limsup _{t \rightarrow \infty} \frac{\ln V(x(t))}{\ln \psi(t)} \leq \pi-\mu \quad \text { a.s. }
$$

Recalling that $V(x)=\sum_{i=1}^{n} c_{i}\left|x_{i}\right|^{p}$ and letting $\pi \rightarrow \pi_{0}$, we obtain

$$
\limsup _{t \rightarrow \infty} \frac{\ln |x(t)|}{\ln \psi(t)} \leq-\frac{\mu-\pi_{0}}{p} \quad \text { a.s. }
$$

as required.

Theorem 3.3 not only provides a systematic treatment for $p$-th moment $\psi$ type stability and almost sure $\psi$-type stability, but also gives conditions that the former implies the latter. To see this, under Assumptions 2.1-2.2 and conditions (3.6)-(3.8), we have conclusions (3.3) and (3.9) which are $p$-th moment $\psi$-type stability and almost sure $\psi$-type stability, respectively. Moreover, taking $\psi(t)=$ $e^{t}$ and $\psi(t)=\left(1+t^{+}\right)$, respectively, we obtain the exponential stability, the polynomial stability and that the exponential stability implies the polynomial stability.

Remark 3.4. If we choose $\psi(t)=e^{t}$, then there exists $\pi_{0}=0 \in[0, \mu)$ such that for any $\pi>\pi_{0}$ condition (3.8) holds. This means that, when $\psi(t)=e^{t}$, condition (3.8) holds automatically. Thus, if condition (3.7) is satisfied, then the $p$ th moment exponential stability can be used to derive the almost sure exponential stability for Eq.(1.1). 


\section{Further results}

Obviously, in Theorem 3.3, it is inconvenient to check condition (3.6) since it is unrelated to both functions $f$ and $g$ of the Eq.(1.1) explicitly. Based on Theorem 3.3, a useful criterion will be established and it can be easily implementable in this section.

To specialize condition (3.6), we impose some conditions on the functions $f$ and $g$ to guarantee Theorem 3.3. For any $(t, x, y) \in \mathbb{R}_{+} \times \mathbb{R}^{n} \times \mathbb{R}^{n}$,

$$
\begin{aligned}
& \begin{array}{l}
\text { (H1) } x_{i} f_{i}(t, x, y) \leq \psi_{1}(t)\left\{-\sigma_{i 0} x_{i}^{2}-\sigma_{i}\left|x_{i}\right|^{\alpha+2}+\sum_{j=1}^{n}\left[\sigma_{i j} x_{j}^{2}+\bar{\sigma}_{i j} y_{j}^{2} \psi^{-\varepsilon}(\delta(t))\right]\right. \\
\left.+\sum_{j=1}^{n} \sum_{k=1}^{K} \sigma_{i j k}\left|x_{j}\right|^{\alpha_{k}+2}\right\} ;
\end{array} \\
& \text { (H2) }\left|g_{i}(t, x, y)\right|^{2} \leq \psi_{1}(t) \sum_{j=1}^{n} \lambda_{i j} x_{j}^{2}
\end{aligned}
$$

where $\varepsilon, \sigma_{i}, \sigma_{i 0}$ for all $i=1, \ldots, n$ are positive constants, and other parameters are all nonnegative.

Condition (H1) is the one-sided polynomial condition on $f$. When $\alpha=0$, this condition will be specialized as the classical one-sided linear growth condition as in [8]. In addition, condition (H2) is the classical linear growth condition on $g$.

Noting that $\psi^{-\varepsilon}(\delta(t))$ is decreasing in $\varepsilon$ and Definition 1.1, we know that if conditions (H1) and (H2) are satisfied, then they will still hold when $\varepsilon$ is replaced by any $\varepsilon^{\prime} \in(0, \varepsilon)$. For condition (H1), without loss of generality, let $0 \leq \alpha_{1}<\alpha_{2}<\cdots<\alpha_{K}<\alpha$.

To proceed, we need a useful lemma.

Lemma 4.1. Let $\alpha, p>0,0 \leq \alpha_{1} \leq \alpha_{2} \leq \cdots \leq \alpha_{K}<\alpha, a>0, b>z=$ $\sum_{k=1}^{K} z_{k}, z_{k} \geq 0(1 \leq k \leq K)$. If

$$
a>z \rho_{1}
$$

where

$$
\rho_{1}:=\left(\alpha-\alpha_{1}\right)\left(\frac{\alpha_{1}^{\alpha_{1}}}{\alpha^{\alpha}}\right)^{\frac{1}{\alpha-\alpha_{1}}},
$$

when $\rho_{1}=0$ if $\alpha_{1}=\alpha$ and $\rho_{1}=1$ if $\alpha_{1}=0$, then there exists a constant $\bar{a} \in(0, a)$ such that for any $t \geq 0$,

$$
a t^{p}+b t^{\alpha+p}-\sum_{k=1}^{K} z_{k} t^{\alpha_{k}+p} \geq \bar{a} t^{p} .
$$


Proof. If we can prove that there is a constant $a_{0} \in\left(z \rho_{1}, a\right)$ such that for any $t \geq 0$

$$
F(t):=a_{0}+b t^{\alpha}-\sum_{k=1}^{K} z_{k} t^{\alpha_{k}} \geq 0,
$$

then we can get the desired assertion. Two cases will be considered.

Case 1: $K=1$. Let $z=z_{1}$ and $\beta=\alpha_{1}$. Then $F(t)=a_{0}+b t^{\alpha}-z t^{\beta}$ for $t \geq 0$. Assume that $\alpha>\beta>0$ (when $\alpha=\beta$, the proof is obvious). It is noted that there exists a unique $t_{0}=(\beta z / \alpha b)^{1 /(\alpha-\beta)}$ such that $F^{\prime}\left(t_{0}\right)=0$. By condition (4.1), recalling that $z<b$ and $a_{0}>z \rho_{1}$, we have

$$
\begin{aligned}
F\left(t_{0}\right) & =a_{0}-\frac{z(\alpha-\beta)}{\alpha}\left(\frac{\beta z}{\alpha b}\right)^{\frac{\beta}{\alpha-\beta}} \\
& =a_{0}-z(\alpha-\beta)\left(\frac{\beta^{\beta}}{\alpha^{\alpha}}\right)^{\frac{1}{\alpha-\beta}}\left(\frac{z}{b}\right)^{\frac{\beta}{\alpha-\beta}} \\
& >0 .
\end{aligned}
$$

Noting that $F(0)=a_{0}, F(\infty)=\infty$, we get for any $t \geq 0, F(t) \geq a_{0} \wedge F\left(t_{0}\right)>0$.

Case 2: $K>1$. Noting that $0 \leq \alpha_{1} \leq \alpha_{2} \leq \cdots \leq \alpha_{K}<\alpha$, we have

$$
F(t) \geq\left\{\begin{array}{l}
a_{0}+b t^{\alpha}-z t^{\alpha_{1}}:=F_{1}(t), \text { if } t \in[0,1] \\
a_{0}+b t^{\alpha}-z t^{\alpha_{K}}:=F_{K}(t), \text { if } t>1
\end{array}\right.
$$

By Case 1 , we know for all $t \geq 0, F_{1}(t) \geq 0$. Let

$$
G(x)=(\alpha-x)\left(\frac{x^{x}}{\alpha^{\alpha}}\right)^{\frac{1}{\alpha-x}}
$$

for $x \in(0, \alpha)$. Direct computation produces that

$$
\frac{G^{\prime}(x)}{G(x)}=\frac{d \log G(x)}{d x}=\frac{\alpha \log (x / \alpha)}{(\alpha-x)^{2}}<0,
$$

which implies that for $x \in(0, \alpha), G(x)$ is decreasing, so we have $a>z G\left(\alpha_{1}\right) \geq$ $z G\left(\alpha_{K}\right)$. By the proof of Case 1, we therefore have that for all $t \geq 0, F_{K}(t) \geq 0$.

In sum, for all $t \geq 0, F(t) \geq F_{1}(t) \wedge F_{K}(t) \geq 0$, as desired. The proof is complete.

To ease our presentation, we introduce the following notations:

$$
\begin{gathered}
\sigma_{i \bullet}=\sum_{j=1}^{n} \sigma_{i j}, \quad \bar{\sigma}_{i \bullet}=\sum_{j=1}^{n} \bar{\sigma}_{i j} \quad \lambda_{i \bullet}=\sum_{j=1}^{n} \lambda_{i j}, \quad D_{i}=\sum_{k=1}^{K} d_{i k} ; \\
\gamma_{i}=p \sigma_{i 0}-(p-2)\left(\sigma_{i \bullet}+\bar{\sigma}_{i \bullet}\right)-\frac{(p-1)(p-2)}{2} \lambda_{i \bullet} ; \\
\zeta_{i}=p(p-2) \rho_{1} \sum_{j=1}^{n} \sum_{k=1}^{K} \frac{\sigma_{i j k}}{\alpha_{k}+p}, \quad \omega_{i}=\left(p \sigma_{i} \rho_{1}\right) \wedge \gamma_{i} ;
\end{gathered}
$$




$$
\Sigma=\left[\sigma_{i j}\right], \quad \Lambda=\left[\lambda_{i j}\right], \quad \Sigma_{k}=\left[\sigma_{i j k} \frac{\alpha_{k}+2}{\alpha_{k}+p}\right], k=1, \ldots, K .
$$

By virtue of the $M$-matrix theory, we will give a new criterion to measure the $\psi$-type stability of Eq.(1.1).

Theorem 4.2. Let conditions (3.8), (H1) and (H2) hold. If for $\omega_{i}, \zeta_{i}, \Sigma, \Lambda$ and $\Sigma_{k}$ defined by (4.4)-(4.5),

$$
Q=\operatorname{diag}\left(\omega_{i}-\zeta_{i}\right)-4 \Sigma-(p-1) \Lambda-\rho_{1} p \sum_{k=1}^{K}\left(\Sigma_{k}\right)
$$

is an $M$-matrix, then for any initial data $\xi \in B C$ and $\mu \in(0, \varepsilon]$, the global solution $x(t, \xi)$ of Eq.(1.1) satisfies (3.2)-(3.4) and (3.9).

Proof. Step 1. $p$-th moment $\psi$-type stability: Since $Q$ is an $M$-matrix, there surely exists $c \in \mathbb{R}_{++}^{n}$ such that $Q^{\mathrm{T}} c \in \mathbb{R}_{++}^{n}$ (See [4]). Applying (2.1) in $V=\sum_{i=1}^{n} c_{i}\left|x_{i}\right|^{p}$ yields

$$
\begin{aligned}
\mathcal{L} V(t, x, y) & =p \sum_{i=1}^{n} c_{i}\left|x_{i}\right|^{p-2} x_{i} f_{i}(t, x, y)+\frac{1}{2} p(p-1) \sum_{i=1}^{n} c_{i}\left|x_{i}\right|^{p-2}\left|g_{i}(t, x, y)\right|^{2} \\
& :=I_{1}+I_{2} .
\end{aligned}
$$

By condition (H1), we first estimate $I_{1}$.

$$
\begin{gathered}
\frac{I_{1}}{\psi_{1}(t)} \leq p \sum_{i=1}^{n} c_{i}\left|x_{i}\right|^{p-2}\left\{-\sigma_{i 0} x_{i}^{2}-\sigma_{i}\left|x_{i}\right|^{\alpha+2}+\sum_{j=1}^{n}\left[\sigma_{i j} x_{j}^{2}+\bar{\sigma}_{i j} y_{j}^{2} \psi^{-\varepsilon}(\delta(t))\right]\right. \\
\left.+\sum_{j=1}^{n} \sum_{k=1}^{K} \sigma_{i j k}\left|x_{j}\right|^{\alpha_{k}+2}\right\} \\
\leq-p \sum_{i=1}^{n} c_{i} \sigma_{i 0}\left|x_{i}\right|^{p}-p \sum_{i=1}^{n} c_{i} \sigma_{i}\left|x_{i}\right|^{\alpha+p}+p \sum_{i, j=1}^{n} c_{i} \sigma_{i j} \frac{(p-2)\left|x_{i}\right|^{p}+2\left|x_{j}\right|^{p}}{p} \\
+p \sum_{i, j=1}^{n} c_{i} \bar{\sigma}_{i j} \frac{(p-2)\left|x_{i}\right|^{p}+2\left|y_{j}\right|^{p}}{p} \psi^{-\varepsilon}(\delta(t)) \\
+p \sum_{i, j=1}^{n} c_{i} \sum_{k=1}^{K} \sigma_{i j k} \frac{(p-2)\left|x_{i}\right|^{\alpha_{k}+p}+\left(\alpha_{k}+2\right)\left|x_{j}\right|^{\alpha_{k}+p}}{\alpha_{k}+p}
\end{gathered}
$$

where we have used the Young inequality

$$
x^{\alpha} y^{\beta} \leq \frac{\alpha|x|^{\alpha+\beta}+\beta|y|^{\alpha+\beta}}{\alpha+\beta}, \quad x, y \geq 0, \alpha, \beta>0 .
$$

Using condition (H2) and the Young inequality again, we have

$$
\frac{I_{2}}{\psi_{1}(t)} \leq \frac{1}{2} p(p-1) \sum_{i=1}^{n} c_{i}\left|x_{i}\right|^{p-2} \sum_{j=1}^{n} \lambda_{i j} x_{j}^{2}
$$




$$
\begin{aligned}
& \leq \frac{1}{2} p(p-1) \sum_{i, j=1}^{n} c_{i} \lambda_{i j} \frac{(p-2)\left|x_{i}\right|^{p}+2\left|x_{j}\right|^{p}}{p} \\
& =\frac{p-1}{2} \sum_{i, j=1}^{n} c_{i} \lambda_{i j}\left[(p-2)\left|x_{i}\right|^{p}+2\left|x_{j}\right|^{p}\right] .
\end{aligned}
$$

Therefore,

$$
\begin{aligned}
& \frac{I_{1}+I_{2}}{\psi_{1}(t)} \\
\leq & -\sum_{i=1}^{n}\left[p c_{i} \sigma_{i 0}-(p-2) c_{i} \sum_{j=1}^{n} \sigma_{i j}-(p-2) c_{i} \sum_{j=1}^{n} \bar{\sigma}_{i j}-2 \sum_{j=1}^{n} c_{j} \sigma_{j i}\right. \\
& \left.\quad \frac{(p-1)(p-2)}{2} c_{i} \sum_{j=1}^{n} \lambda_{i j}-(p-1) \sum_{j=1}^{n} c_{j} \lambda_{j i}\right]\left|x_{i}\right|^{p} \\
& -p \sum_{i=1}^{n} c_{i} \sigma_{i}\left|x_{i}\right|^{\alpha+p}+2 \sum_{i, j=1}^{n} c_{i} \bar{\sigma}_{i j}\left|y_{j}\right|^{p} \psi^{-\varepsilon}(\delta(t)) \\
& +p \sum_{i, j=1}^{n} \sum_{k=1}^{K}\left[(p-2) c_{i} \sigma_{i j k} \frac{1}{\alpha_{k}+p}+c_{j} \sigma_{j i k} \frac{\alpha_{k}+2}{\alpha_{k}+p}\right]\left|x_{i}\right|^{\alpha_{k}+p} \\
:= & -\sum_{i=1}^{n}\left[a_{i}\left|x_{i}\right|^{p}+b_{i}\left|x_{i}\right|^{\alpha+p}-\sum_{k=1}^{K} d_{i k}\left|x_{i}\right|^{\alpha_{k}+p}\right]+2 \sum_{i, j=1}^{n} c_{i} \bar{\sigma}_{i j}\left|y_{j}\right|^{p} \psi^{-\varepsilon}(\delta(t)),
\end{aligned}
$$

where

$$
\begin{aligned}
a_{i}= & p c_{i} \sigma_{i 0}-(p-2) c_{i}\left(\sigma_{i \bullet}+\bar{\sigma}_{i \bullet}\right)-\frac{(p-1)(p-2)}{2} c_{i} \lambda_{i \bullet} \\
& -\sum_{j=1}^{n} c_{j}\left[2 \sigma_{j i}+(p-1) \lambda_{j i}\right] ; \\
b_{i}= & p c_{i} \sigma_{i} ; \\
d_{i k}= & p\left[(p-2) c_{i} \sum_{j=1}^{n} \frac{\sigma_{i j k}}{\alpha_{k}+p}+\sum_{j=1}^{n} c_{j} \sigma_{j i k} \frac{\alpha_{k}+2}{\alpha_{k}+p}\right] .
\end{aligned}
$$

Hence, we obtain

$$
\begin{aligned}
& a_{i}-\rho_{1} D_{i} \\
= & c_{i}\left[p \sigma_{i 0}-(p-2)\left(\sigma_{i \bullet}+\bar{\sigma}_{i \bullet}\right)-\frac{(p-1)(p-2)}{2} \lambda_{i \bullet}-p(p-2) \rho_{1} \sum_{j=1}^{n} \sum_{k=1}^{K} \frac{\sigma_{i j k}}{\alpha_{k}+p}\right] \\
& -\sum_{j=1}^{n} c_{j}\left[2 \sigma_{j i}+(p-1) \lambda_{j i}\right]-p \rho_{1} \sum_{j=1}^{n} \sum_{k=1}^{K} c_{j} \sigma_{j i k} \frac{\alpha_{k}+2}{\alpha_{k}+p} ;
\end{aligned}
$$




$$
\begin{aligned}
& b_{i}-D_{i} \\
= & c_{i}\left[p \sigma_{i}-p(p-2) \sum_{j=1}^{n} \sum_{k=1}^{K} \frac{\sigma_{i j k}}{\alpha_{k}+p}\right]-p \sum_{j=1}^{n} \sum_{k=1}^{K} c_{j} \sigma_{j i k} \frac{\alpha_{k}+2}{\alpha_{k}+p} .
\end{aligned}
$$

Noting that $Q^{\mathrm{T}} c \in \mathbb{R}_{++}^{n}$ implies that for all $i=1, \ldots, n, a_{i}>\rho_{1} D_{i}, b_{i}>D_{i}$. Thus, all the conditions of Lemma 4.1 are tested, so by the proof of Lemma 4.1 , we observe that there exists a constant $\bar{a}_{i} \in\left(0, a_{i}-\rho_{1} D_{i}\right)$ such that

$$
a_{i}\left|x_{i}\right|^{p}+b_{i}\left|x_{i}\right|^{\alpha+p}-\sum_{k=1}^{K} d_{i k}\left|x_{i}\right|^{\alpha_{k}+p} \geq \bar{a}_{i}\left|x_{i}\right|^{p} .
$$

This implies that

$$
\frac{I_{1}+I_{2}}{\psi_{1}(t)} \leq-\sum_{i=1}^{n} \bar{a}_{i}\left|x_{i}\right|^{p}+2 \sum_{i, j=1}^{n} c_{i} \bar{\sigma}_{i j}\left|y_{j}\right|^{p} \psi^{-\varepsilon}(\delta(t)),
$$

that is

$$
\begin{aligned}
\mathcal{L} V(t, x, y) & \leq \psi_{1}(t)\left[-\sum_{i=1}^{n} \bar{a}_{i}\left|x_{i}\right|^{p}+2 \sum_{i, j=1}^{n} c_{i} \bar{\sigma}_{i j}\left|y_{j}\right|^{p} \psi^{-\varepsilon}(\delta(t))\right] \\
& \leq \psi_{1}(t)\left[-\sum_{i=1}^{n} \bar{a}_{i}\left|x_{i}\right|^{p}+2 \sum_{i=1}^{n}\left(\sum_{j=1}^{n} c_{j} \bar{\sigma}_{j i}\right)\left|y_{i}\right|^{p} \psi^{-\varepsilon}(\delta(t))\right] .
\end{aligned}
$$

Next, we prove that (4.8) implies condition (3.6). Noting that $Q^{\mathrm{T}} c \in \mathbb{R}_{++}^{n}$ implies that $2 \sum_{j=1}^{n} c_{j} \sigma_{j i}<a_{i}-D_{i} \rho_{1}$, by the proof of Lemma 4.1, we observe that there exists a constant $\bar{a}_{i} \in\left(2 \sum_{j=1}^{n} c_{j} \sigma_{j i}, a_{i}-D_{i} \rho_{1}\right)$ such that (4.7) is satisfied and $\bar{a}_{i}>2 \sum_{j=1}^{n} c_{j} \sigma_{j i}$. This implies $\bar{a}_{i} c_{i}^{-1}>2 c_{i}^{-1} \sum_{j=1}^{n} c_{j} \sigma_{j i}$. So there must exist positive constants $a, b$ such that

$$
\left\{\begin{array}{l}
a<\bar{a}_{i} c_{i}^{-1} \\
b \geq 2 c_{i}^{-1} \sum_{j=1}^{n} c_{j} \sigma_{j i}, \\
a>b .
\end{array}\right.
$$

Therefore, we have

$$
\begin{aligned}
\mathcal{L} V(t, x, y) & \leq \psi_{1}(t)\left[-a \sum_{i=1}^{n} c_{i}\left|x_{i}\right|^{p}+b \sum_{i=1}^{n} c_{i}\left|y_{i}\right|^{p} \psi^{-\varepsilon}(\delta(t))\right] \\
& =\psi_{1}(t)\left[-a V(x)+b V(y) \psi^{-\varepsilon}(\delta(t))\right] .
\end{aligned}
$$

That is, condition (3.6) of Theorem 3.3 is tested. Under conditions (H1) and (H2), we confirm the existence of (2.4). Then the required assertions (3.2)-(3.4) hold following Theorem 3.3. 
Step 2. Almost sure $\psi$-type stability: For the function $V(x)=\sum_{i=1}^{n} c_{i}\left|x_{i}\right|^{p}$, we have $V_{x}(x)=p \sum_{i=1}^{n} c_{i}\left|x_{i}\right|^{p-2} x_{i}$. Using condition (H2) and noting $\left|\psi_{1}(t)\right| \leq$ $\phi$, we compute

$$
\begin{aligned}
\left|V_{x}(x) g(t, x, y)\right|^{2} & \leq\left|V_{x}(x)\right|^{2}|g(t, x, y)|^{2} \\
& \leq\left.\left. p^{2}\left|\sum_{i=1}^{n} c_{i}\right| x_{i}\right|^{p-2} x_{i}\right|^{2}\left|\psi_{1}(t)\right| \sum_{j=1}^{n} \lambda_{i j} x_{j}^{2} \\
& \leq\left.\left. p^{2} \phi\left|\sum_{i=1}^{n} c_{i}\right| x_{i}\right|^{p-1}\right|^{2} \sum_{j=1}^{n} \lambda_{i j} x_{j}^{2} .
\end{aligned}
$$

Using the elementary inequality

$$
\left(\sum_{j=1}^{n} \lambda_{j} x_{j}\right)^{2} \leq \sum_{j=1}^{n} \lambda_{j} \sum_{j=1}^{n} \lambda_{j} x_{j}^{2}, \quad \lambda_{j} \geq 0, x_{j} \in \mathbb{R}
$$

we have

$$
\begin{aligned}
\left|V_{x}(x) g(t, x, y)\right|^{2} & \leq p^{2} \phi \sum_{i=1}^{n} c_{i} \sum_{i=1}^{n} c_{i}\left|x_{i}\right|^{2 p-2} \sum_{j=1}^{n} \lambda_{i j} x_{j}^{2} \\
& \leq p^{2} \phi \sum_{i=1}^{n} c_{i} \sum_{i, j=1}^{n} c_{i} \lambda_{i j} \frac{(2 p-2)\left|x_{i}\right|^{2 p}+2\left|x_{j}\right|^{2 p}}{2 p} \\
& =p \phi \sum_{i=1}^{n} c_{i} \sum_{i, j=1}^{n} c_{i} \lambda_{i j}\left[(p-1) x_{i}^{2 p}+x_{j}^{2 p}\right] .
\end{aligned}
$$

Then we claim that there must exist some constant such that

$$
\left|V_{x}(x) g(t, x, y)\right|^{2} \leq C V(x)^{2} .
$$

Therefore, condition (3.7) of Theorem 3.3 is tested. Making use of conditions (3.7) and (3.8), we have assertion (3.9), as desired.

By comparing Theorem 3.3 with Theorem 4.2, one finds that the former is more general while the later is much simpler. Somewhat remarkably, Theorem 4.2 merely requires the matrix $Q$ to be an $M$-matrix, which will bring more convenience in applications. Moreover, by modifying slightly condition (4.6) in Theorem 4.2, we have another useful theorem.

Define

$$
\begin{aligned}
& M=\operatorname{diag}\left(p \sigma_{i} \rho_{1}-\zeta_{i}\right)-p \rho_{1} \sum_{k=1}^{K}\left(\Sigma_{k}\right), \\
& N=\operatorname{diag}\left(\gamma_{i}-\zeta_{i}\right)-2 \Sigma-(p-1) \Lambda-p \rho_{1} \sum_{k=1}^{K}\left(\Sigma_{k}\right),
\end{aligned}
$$




$$
H=\operatorname{diag}\left(\gamma_{i}-\zeta_{i}\right)-4 \Sigma-(p-1) \Lambda-p \rho_{1} \sum_{k=1}^{K}\left(\Sigma_{k}\right),
$$

where $\gamma_{i}, \zeta_{i}, \Sigma, \Lambda$ and $\Sigma_{k}$ are defined by(4.4)-(4.5).

Theorem 4.3. Under conditions (3.8), (H1) and (H2). If there exists $c=$ $\left(c_{1}, \ldots, c_{n}\right)^{\mathrm{T}} \in \mathbb{R}_{++}^{n}$ such that $M^{\mathrm{T}} c, N^{\mathrm{T}} c, H^{\mathrm{T}} c \in \mathbb{R}_{++}^{n}$, then the conclusions of Theorem 4.2 hold.

Proof. Recalling the proof of Theorem 4.2 and noting that there exists $c=$ $\left(c_{1}, \ldots, c_{n}\right)^{\mathrm{T}} \in \mathbb{R}_{++}^{n}$ such that $M^{\mathrm{T}} c, N^{\mathrm{T}} c, H^{\mathrm{T}} c \in \mathbb{R}_{++}^{n}$, we get

$$
\begin{gathered}
c_{i}\left(p \sigma_{i} \rho_{1}-\zeta_{i}\right)-p \rho_{1} \sum_{j=1}^{n} \sum_{k=1}^{K} c_{j} \sigma_{j i k} \frac{\alpha_{k}+2}{\alpha_{k}+p}>0 \\
c_{i}\left(\gamma_{i}-\zeta_{i}\right)-\sum_{j=1}^{n} c_{j}\left[2 \sigma_{j i}+(p-1) \lambda_{j i}\right]-p \rho_{1} \sum_{j=1}^{n} \sum_{k=1}^{K} c_{j} \sigma_{j i k} \frac{\alpha_{k}+2}{\alpha_{k}+p}>0 \\
c_{i}\left(\gamma_{i}-\zeta_{i}\right)-\sum_{j=1}^{n} c_{j}\left[4 \sigma_{j i}+(p-1) \lambda_{j i}\right]-p \rho_{1} \sum_{j=1}^{n} \sum_{k=1}^{K} c_{j} \sigma_{j i k} \frac{\alpha_{k}+2}{\alpha_{k}+p}>0
\end{gathered}
$$

which implies $a_{i}>D_{i} \rho_{1}, b_{i}>D_{i}$ and $a_{i}-D_{i} \rho_{1}>2 \sum_{j=1}^{n} c_{j} \sigma_{j i}$. Repeating the proof of Theorem 4.2 yields the desired assertion.

It is easy to see that the conditions in Theorem 4.2 are stronger than those of Theorem 4.3, while the latter gains more efficiency in applications. For the applications of Theorem 4.3, is there surely a $c \in \mathbb{R}_{++}^{n}$ such that $M^{\mathrm{T}} c, N^{\mathrm{T}} c, H^{\mathrm{T}} c \in \mathbb{R}_{++}^{n}$ ? The following lemma presents a positive answer to this question and provides a sufficient condition.

Lemma 4.4. Assume that $A_{k} \in \mathbb{R}^{n \times n}(1 \leq k \leq K)$, if $A_{1}, \ldots, A_{K-1}, A_{k} A_{l}^{-1}$ $(1 \leq l<k \leq K)$ are all $M$-matrices, then there exists $c \in \mathbb{R}_{++}^{n}$ such that $A_{k} c \in \mathbb{R}_{++}^{n}(1 \leq k \leq K)$.

Proof. When $k=1$, the result holds clearly.

Suppose $K>1$. If $A_{1}, \ldots, A_{K-1}, A_{k} A_{l}^{-1}(1 \leq l<k \leq K)$ are $M$-matrices, then we have

$$
A_{2} A_{1}^{-1}, \ldots, A_{K-1} A_{1}^{-1}, A_{k} A_{1}^{-1}\left(A_{l} A_{1}^{-1}\right)^{-1}(2 \leq l<k \leq K)
$$

are $M$-matrices. By induction, there exists $c^{\prime} \in \mathbb{R}_{++}^{n}$ such that

$$
A_{k} A_{1}^{-1} c^{\prime} \in \mathbb{R}_{++}^{n}, \quad 2 \leq k \leq K .
$$

Let $c=A_{1}^{-1} c^{\prime}$, by $A_{1}^{-1} \geq 0$, we derive that there exists $c \in \mathbb{R}_{++}^{n}$, such that $A_{k} c \in \mathbb{R}_{++}^{n}(1 \leq k \leq K)$. This completes the proof. 
By Lemma 4.4, we observe if we show $M, N, H^{-1} M, H^{-1} N$ and $N^{-1} M$ are all $M$-matrices, then we will be sure that there exists $c \in \mathbb{R}_{++}^{n}$ such that $M^{\mathrm{T}} c, N^{\mathrm{T}} c, H^{\mathrm{T}} c \in \mathbb{R}_{++}^{n}$. However, this criterion is only sufficient but not necessary. Therefore, the testification of the criterion of Theorem 4.3 is more complicated than that of Theorem 4.2.

Furthermore, noting the expressions of the matrices $N$ and $H$ in Theorem 4.2 , we see that if $H$ is an $M$-matrix, then $N$ must be. Therefore, we have the following corollary.

Corollary 4.5. Under conditions (3.8), (H1) and (H2). If there exists $c \in \mathbb{R}_{++}^{n}$ such that $M^{\mathrm{T}} c, H^{\mathrm{T}} c \in \mathbb{R}_{++}^{n}$, then the conclusions of Theorem 4.3 hold.

For the easy use of Corollary 4.5, let us cite some useful results on $M$ matrices in Berman and Plemmons's book [4].

Definition 4.1. Assume that $A=\left[a_{i j}\right] \in \mathbb{R}^{n \times n}, a_{i j} \leq 0<a_{i i}(i \neq j, i, j=$ $1, \ldots, n)$, then the following three terms are equivalent:

(i) $A$ is an $M$-matrix;

(ii) there exists $c \in \mathbb{R}_{++}^{n}$ such that $A c \in \mathbb{R}_{++}^{n}$;

(iii) All the leading principal minors of $A$ are positive.

In particular, for $2 \times 2$ matrices, we have the following accurate criterion.

Lemma 4.6. Let $M=\left[m_{i j}\right] \in \mathbb{R}^{2 \times 2}, H=\left[h_{i j}\right] \in \mathbb{R}^{2 \times 2}, m_{i i}, h_{i i}>0, m_{i j}, h_{i j} \leq$ 0 for $i \neq j, i, j=1,2$. Then there exists $c \in \mathbb{R}_{++}^{2}$ such that $M^{\mathrm{T}} c, H^{\mathrm{T}} c \gg 0$ if and only if

$$
\frac{\left|m_{12}\right|}{m_{22}} \vee \frac{\left|h_{12}\right|}{h_{22}}<\frac{m_{11}}{\left|m_{21}\right|} \wedge \frac{h_{11}}{\left|h_{21}\right|}
$$

Proof. If there exists $c \in \mathbb{R}_{++}^{2}$ such that $M^{\mathrm{T}} c, H^{\mathrm{T}} c \gg 0$, then it follows from inequalities $m_{1 i} c_{1}+m_{2 i} c_{2}>0$ and $h_{1 i} c_{1}+h_{2 i} c_{2}>0(i=1,2)$ that

$$
\frac{\left|m_{12}\right|}{m_{22}}<\frac{c_{2}}{c_{1}}<\frac{m_{11}}{\left|m_{21}\right|} \quad \text { and } \quad \frac{\left|h_{12}\right|}{h_{22}}<\frac{c_{2}}{c_{1}}<\frac{h_{11}}{\left|h_{21}\right|} \text {, }
$$

which shows (4.16). On the other hand, if (4.16) holds, choose

$$
c_{2} \in\left(\frac{\left|m_{12}\right|}{m_{22}} \vee \frac{\left|h_{12}\right|}{h_{22}}, \frac{m_{11}}{\left|m_{21}\right|} \wedge \frac{h_{11}}{\left|h_{21}\right|}\right) .
$$

Let $c_{1}=1$, so that $c=\left(c_{1}, c_{2}\right)^{\mathrm{T}} \in \mathbb{R}_{++}^{2}$ satisfies $M^{\mathrm{T}} c, H^{\mathrm{T}} c \gg 0$.

Remark 4.7. Lemma 4.6 indicates that Corollary 4.5 can be easily implementable in a two-dimensional case. To see this, if condition (4.16) is satisfied, there must exist a $c=\left(c_{1}, c_{2}\right)^{\mathrm{T}} \in \mathbb{R}_{++}^{2}$ such that $M^{\mathrm{T}} c, H^{\mathrm{T}} c \in \mathbb{R}_{++}^{2}$. However, in the case of dimension higher than two, it is more difficult to find such a $c \in \mathbb{R}_{++}^{n}$, but Theorem 4.2 finds its way if $Q$ proves to be an $M$-matrix. 


\section{Examples}

In this section, we shall consider several two-dimensional equations to illustrate our results.

\section{Example 5.1. Consider}

$$
\begin{aligned}
d x_{i}(t)= & \psi_{1}(t)\left\{-5 x_{i}(t)-b x_{i}^{3}(t)+\left[y_{1}(t)+y_{2}(t)\right] \psi^{-\varepsilon}(\delta(t))+x_{j}^{2}(t)\right\} d t \\
& +x_{i}(t) \sqrt{\psi_{1}(t)} d w(t)
\end{aligned}
$$

where $i, j=1,2, b>0$ is a constant, $\psi_{1}(t)$ is defined in Definition 1.1 and $y_{i}(t)=x_{i}(t-\delta(t)), \delta(t) \in C^{1}\left(\mathbb{R}_{+}, \mathbb{R}_{+}\right)$.

Let $\tau_{0}=\delta(0)$. Then $t-\delta(t) \in\left[-\tau_{0}, \infty\right)$. If $\tau_{0}<\infty$, then $\psi^{-\varepsilon}(\delta(t)) \geq$ $\psi^{-\varepsilon}\left(\tau_{0}\right) \rightarrow 1(\varepsilon \rightarrow 0)$, so $\psi^{-\varepsilon}(\delta(t))$ can be deleted when $\tau_{0}<\infty$, that is, $\psi^{-\varepsilon}(\delta(t))$ works only when delay is unbounded. Furthermore, if we choose $\psi(t)=e^{t}$, then $\psi_{1}(t)=1$ and Eq.(5.1) becomes the following special case

$$
\begin{aligned}
d x_{i}(t)= & \left\{-5 x_{i}(t)-b x_{i}^{3}(t)+x_{1}(t-\delta(t))+x_{2}(t-\delta(t))+x_{j}^{2}(t)\right\} d t \\
& +x_{i}(t) d w(t) .
\end{aligned}
$$

As for Eq. (5.1), on one hand, we compute

$$
\begin{aligned}
& \frac{x_{i} f_{i}(t, x, y)}{\psi_{1}(t)} \\
= & -5 x_{i}^{2}-b x_{i}^{4}+\left(x_{i} y_{1}+x_{i} y_{2}\right) \psi^{-\varepsilon}(\delta(t))+x_{i} x_{j}^{2} \\
\leq & -5 x_{i}^{2}-b x_{i}^{4}+\frac{x_{i}^{2}+y_{1}^{2} \psi^{-\varepsilon}(\delta(t))}{2}+\frac{x_{i}^{2}+y_{2}^{2} \psi^{-\varepsilon}(\delta(t))}{2}+\frac{x_{i}^{3}+2 x_{j}^{2}}{3} \\
= & -5 x_{i}^{2}-b x_{i}^{4}+\left[x_{i}^{2}+\frac{1}{2}\left(y_{1}^{2}+y_{2}^{2}\right) \psi^{-\varepsilon}(\delta(t))\right]+\frac{1}{3}\left(x_{i}^{3}+2 x_{j}^{3}\right),
\end{aligned}
$$

which shows that condition (H1) holds with $\sigma_{i 0}=5, \sigma_{i}=b, \sigma_{i i}=1, \sigma_{i j}=0$, $\bar{\sigma}_{i 1}=\bar{\sigma}_{i 2}=1 / 2, \sigma_{i i 1}=1 / 3, \sigma_{i j 1}=2 / 3, K=1, \alpha_{k}=1, \alpha=2$. On the other hand,

$$
\left|g_{i}(t, x, y)\right|^{2}=\psi_{1}(t) x_{i}^{2}(t),
$$

which states that condition (H2) holds with $\lambda_{i i}=1, \lambda_{i j}=0$.

Choosing $p=2$ and substituting above parameters into (4.2), (4.4) and (4.5) give $\rho_{1}=1 / 4, \sigma_{i} \bullet=1, \bar{\sigma}_{i \bullet}=1, \lambda_{i \bullet}=1, \gamma_{i}=10, \zeta_{i}=0, \omega_{i}=(b / 2) \wedge 10$, and

$$
\Sigma=\left(\begin{array}{ll}
1 & 0 \\
0 & 1
\end{array}\right), \quad \Lambda=\left(\begin{array}{cc}
1 & 0 \\
0 & 1
\end{array}\right), \quad \Sigma_{1}=\left(\begin{array}{cc}
1 / 3 & 2 / 3 \\
2 / 3 & 1 / 3
\end{array}\right) .
$$

Next, the substitution of above parameters into (4.6) implies (4.10) and (4.12) yields

$$
\begin{gathered}
Q=\left(q_{i j}\right)_{2 \times 2}=\left(\begin{array}{cc}
\frac{b}{2} \wedge 10-\frac{31}{6} & -\frac{1}{3} \\
-\frac{1}{3} & \frac{b}{2} \wedge 10-\frac{31}{6}
\end{array}\right), \\
M=\left(m_{i j}\right)_{2 \times 2}=\left(\begin{array}{cc}
\frac{b}{2}-\frac{1}{6} & -\frac{1}{3} \\
-\frac{1}{3} & \frac{b}{2}-\frac{1}{6}
\end{array}\right)
\end{gathered}
$$




$$
H=\left(h_{i j}\right)_{2 \times 2}=\left(\begin{array}{cc}
29 / 6 & -1 / 3 \\
-1 / 3 & 29 / 6
\end{array}\right) .
$$

By Definition 4.1, $Q$ is an $M$-matrix if and only if $q_{11}>0, q_{11} q_{22}>q_{12} q_{21}$, that is, $[(b / 2) \wedge 10]-(31 / 6)>1 / 3$, or $b>11$. Therefore, by Theorem 4.2 , we have that if $b>11$, the trivial solution of Eq.(5.1) is $\psi$-type stable in mean square. Furthermore, if there exists $\pi_{0} \in[0, \mu)$ such that (3.8) is satisfied, then the trivial solution of Eq.(5.1) is almost surely $\psi$-type stable.

Substituting $m_{i j}$ and $h_{i j}(i, j=1,2)$ into inequality (4.16), we obtain $\frac{2}{3 b-1} \vee$ $\frac{2}{29}<\frac{3 b-1}{2} \wedge \frac{29}{2}$, which implies that $b>10$. So, using Corollary 4.5, we obtain that if $b>10$, the trivial solution of Eq.(5.1) is $\psi$-type stable in mean square. Furthermore, if there exists $\pi_{0} \in[0, \mu)$ such that (3.8) is satisfied, then the trivial solution of Eq.(5.1) is almost surely $\psi$-type stable. It is evident that $b>11$ implies $b>10$, which clearly shows that the conditions of Corollary 4.5 are weaker than those of Theorem 4.2.

Especially, if we choose $\psi(t)=e^{t}$ and let $\pi_{0}=0 \in[0, \mu)$, then $\psi_{1}(t)=1$ and for any $\pi>0, \sum_{k=1}^{\infty} e^{-k \pi}<\infty$. We can conclude that when $b>10$, the trivial solution of Eq.(5.1) is exponentially stable in mean square and almost surely exponentially stable.

Example 5.2. Consider

$$
\left\{\begin{array}{l}
d x_{1}(t)=\left[-7 x_{1}(t)-7 x_{1}^{3}(t)+y_{1}(t) e^{-\varepsilon \delta(t)}\right] d t+\left[x_{1}(t)+\sin ^{2} x_{2}(t)\right] d w(t) ; \\
d x_{2}(t)=\left[-7 x_{2}(t)-7 x_{2}^{3}(t)+y_{2}(t) e^{-\varepsilon \delta(t)}\right] d t+\left[x_{2}(t) \cos x_{1}(t)\right] d w(t),
\end{array}\right.
$$

with initial data $\xi \in B C$, where $y_{i}(t)=x_{i}(t-\delta(t))(i=1,2), \delta(t) \in C^{1}\left(\mathbb{R}_{+}, \mathbb{R}_{+}\right)$.

Define

$$
\begin{aligned}
& f_{1}(t, x, y)=-7 x_{1}-7 x_{1}^{3}+y_{1} e^{-\varepsilon \delta(t)} ; \\
& f_{2}(t, x, y)=-7 x_{2}-7 x_{2}^{3}+y_{2} e^{-\varepsilon \delta(t)} \\
& g_{1}(t, x, y)=x_{1}+\sin ^{2} x_{2} ; \\
& g_{2}(t, x, y)=x_{2} \cos x_{1} .
\end{aligned}
$$

For $i=1,2$, we compute

$$
\begin{aligned}
x_{i} f_{i}(t, x, y) & =-7 x_{i}^{2}-7 x_{i}^{4}+x_{i} y_{i} e^{-\varepsilon \delta(t)} \\
& \leq-7 x_{i}^{2}-7 x_{i}^{4}+\frac{1}{2}\left(x_{i}^{2}+y_{i}^{2} e^{-\varepsilon \delta(t)}\right) \\
\left|g_{1}(t, x, y)\right|^{2} & \leq 2\left(x_{1}^{2}+x_{2}^{2}\right) \\
\left|g_{2}(t, x, y)\right|^{2} & \leq x_{2}^{2} .
\end{aligned}
$$

Choosing $V(x)=\sum_{i=1}^{2} x_{i}^{2}$, we obtain

$$
\mathcal{L} V(t, x, y)=2 \sum_{i=1}^{2}\left(x_{i} f_{i}(t, x, y)+\frac{1}{2}\left|g_{i}(t, x, y)\right|^{2}\right)
$$




$$
\begin{aligned}
& =2 \sum_{i=1}^{2} x_{i} f_{i}(t, x, y)+\sum_{i=1}^{2}\left|g_{i}(t, x, y)\right|^{2} \\
& \leq-11 x_{1}^{2}-14 x_{1}^{4}+y_{1}^{2} e^{-\varepsilon \delta(t)}-10 x_{2}^{2}-14 x_{2} 1^{4}+y_{2}^{2} e^{-\varepsilon \delta(t)} \\
& \leq-10\left(x_{1}^{2}+x_{2}^{2}\right)+\left(y_{1}^{2}+y_{2}^{2}\right) e^{-\varepsilon \delta(t)} .
\end{aligned}
$$

Choosing $a=10>b=1$ shows that condition (3.6) is satisfied.

$$
\begin{aligned}
\left|V_{x}(x) g(t, x, y)\right| & \leq 2 \sum_{i=1}^{2}\left|x_{i}\right|\left(2 x_{1}^{2}+3 x_{2}^{2}\right)^{\frac{1}{2}} \\
& \leq 4\left(x_{1}+x_{2}\right)^{2} \leq 8 V(x),
\end{aligned}
$$

which shows that condition (3.7) is satisfied. Since $\psi(t)=e^{t}$, by Remark 3.4 we know condition (3.8) holds. Applying Theorem 3.3 we have that, for any initial data $\xi \in B C$, the trivial solution of Eq.(5.3) are both exponentially stable in mean square and almost surely exponentially stable.

\section{Conclusions}

In this paper, our efforts focus on obtaining the Razumikhin-type theorems in the unbounded delay case and to develop new techniques on the general decay stability. A Razumikhin-type theorem is first established to obtain the moment stability but without almost sure stability of nonlinear stochastic differential equations with unbounded delay. Then an improved edition can be used to derive not only the moment stability but also the almost sure stability, while the earlier Razumikhin-type theorems solely aim at the moment stability. By virtue of the $M$-matrix techniques, we further develop the aforementioned Razumikhin-type theorems to be easily implementable. An example is given for illustration.

In contrast to the earlier publications in the direction, our emphasis is put more on methodic consideration than on theoretic development, and our contributions are simply summarized as follow: First, existing Razumikhin-type theorems are appropriate only for the bounded delay case, while ours are valid in both the bounded delay and the unbounded delay: Second, existing Razumikhin-type theorems aim at only the moment stability, while ours can be used to obtain not only the moment stability but also the almost sure stability: Third, by virtue of $M$-matrix theory, we develop the Razumikhin-type method to be easily implementable: Last, several kinds of stability, such as the exponential stability and the polynomial stability, can be simultaneously treated in our theory.

Acknowledgement. This Research was supported by the NNSF of China under Grant No. 10871077 and the fundamental research funds for the central universities under Grant No. 2010MS130. 


\section{References}

[1] J. A. D Appleby, Decay and growth rates of solutions of scalar stochastic delay differential equations with unbounded delay and state dependent noise, Stoch. Dyn. 5 (2005), no. $2,133-147$.

[2] J. A. D. Appleby and E. Buckwar, Sufficient condition for polynomial asymptotic behavier of stochastic pantograph equations, available at www.dcu.ie/maths/research/preprint.shtml.

[3] L. Arnold, Stochastic Differential Equations: Theory and applications, Wiley, New York, 1974.

[4] A. Berman and R. J. Plemmons, Nonnegative Matrices in the Mathematical Sciences, SIAM, Philadelphia, PA. 1994.

[5] J. Čermák, The asymptotics of solutions for a class of delay differential equations, Rocky Mountain J. Math. 33 (2003), no. 3, 775-786.

[6] J. Črmák and Stanislava Dvořáková, Asymptotic estimation for some nonlinear delay differential equations, Recults Math. 51 (2008), no. 3-4, 201-213.

[7] M. L. Heard, A change of variables for functional differential equations, J. Differential Equations 18 (1975), 1-10.

[8] D. J. Higham, X. Mao, and C. Yuan, Almost sure and moment exponential stability in the numerical simulation of stochastic differential equations, SIAM J. Numer. Anal. 45 (2007), no. 2, 592-609.

[9] A. Iserles, On the generalized pantograph functional-differential equation, European J. Appl. Math. 4 (1993), no. 1, 1-38.

[10] R. Z. Khas'minskii, Stochastic Stability of Differential Equations, Sijthoff and Noordhoff, 1981.

[11] V. B. Kolmanovskii and V. R. Nosov, Stability of Functional Differential Equations, Academic Press, New York, 1986.

[12] G. Makay and J. Terjéki, On the asymptotic behavior of the pantograph equations, Electron. J. Qual. Theory Differ. Equ. 1998 (1998), no. 2, 1-12.

[13] X. Mao, Exponential Stability of Stochastic Differential Equations, Dekker, 1994.

[14] _ Razumikhin-type theorems on exponential stability of stochastic functional differential equations, Stochastic Process, Stochastic Process. Appl. 65 (1996), no. 2, 233250.

[15] _ Razumikhin-type theorems on exponential stability of neutral stochastic functional differential equations, SIAM J. Math. Anal. 28 (1997), no. 2, 389-401.

[16] Stochastic Differential Equations and Applications, Horwood, Chichester, 1997.

[17] _ A note on the LaSalle-type theorems for stochastic differential delay equations, J. Math. Anal. Appl. 268 (2002), no. 1, 125-142.

[18] _ LaSalle-type theorems for stochastic differential delay equations, J. Math. Anal. Appl. 236 (1999), no. 2, 350-369.

[19] _ The LaSalle-type theorems for stochastic functional differential equations, Nonlinear Stud. 7 (2000), no. 2, 307-328.

[20] _ Stability of Stochastic Differential Equations with Respect to Semimartingales, Longman Scientific and technical, 1991.

[21] _ Stability and stabilisation of stochastic differential delay equations, IET Control Theory Appl. 1 (2007), no. 6, 1551-1566.

[22] X. Mao, A. Matasov, and A. Piunovskiy, Stochastic differential delay equations with Markovian switching, Bernoulli 6 (2000), no. 1, 73-90.

[23] X. Mao and M. J. Rassias, Khasminskii-type theorems for stochastic differential delay equations, Stoch. Anal. Appl. 23 (2005), no. 5, 1045-1069.

[24] X. Mao and C. Yuan, Stochastic Differential Equations with Markovian Switching, Imperial College press, 2006 
[25] Y. Shen, Q. Luo, and X. Mao, The improved LaSalle-type theorems for stochastic functional differential equations, J. Math. Anal. Appl. 318 (2006), no. 1, 134-154.

Xuejing Meng

Department of Statistics and Mathematics

Hubei University of ECONOMics

Wuhan, Hubei 430205, P. R. China

E-mail address: mengxuejing@gmail.com

BAOJIAN YIN

School of Mathematics and Statistics

Huazhong University of Science and Technology

Wuhan, Hubei 430074, P. R. China

E-mail address: yin.bj@mail.hust.edu.cn 